\title{
The role of DNA microarrays in Toxoplasma gondii research, the causative agent of ocular toxoplasmosis
}

\author{
Kevin M. Brown • Ira J. Blader
}

Received: 27 September 2009 / Accepted: 6 November 2009/Published online: 12 December 2009

(C) The Author(s) 2009. This article is published with open access at Springerlink.com

\begin{abstract}
Ocular toxoplasmosis, which is caused by the protozoan parasite Toxoplasma gondii, is the leading cause of retinochoroiditis. Toxoplasma is an obligate intracellular pathogen that replicates within a parasitophorous vacuole. Infections are initiated by digestion of parasites deposited in cat feces or in undercooked meat. Parasites then disseminate to target tissues that include the retina where they then develop into long-lived asymptomatic tissue cysts. Occasionally, cysts reactivate and growth of newly emerged parasites must be controlled by the host's immune system or disease will occur. The mechanisms by which Toxoplasma grows within its host cell, encysts, and interacts with the host's immune system are important questions. Here, we will discuss how the use of DNA microarrays in transcriptional profiling, genotyping, and epigenetic experiments has impacted our understanding of these processes. Finally, we will discuss how these advances relate to ocular toxoplasmosis and how future research on ocular toxoplasmosis can benefit from DNA microarrays.
\end{abstract}

Keywords Microarrays · Uveitis · Retinitis · Parasitology · Host-pathogen interaction $\cdot$ Bioinformatics

\section{Introduction}

Toxoplasma gondii is an obligate intracellular Apicomplexan parasite that can infect a wide range of warm-

K. M. Brown · I. J. Blader $(\bowtie)$

Department of Microbiology and Immunology,

University of Oklahoma Health Sciences Center,

Oklahoma City, OK 73034, USA

e-mail: iblader@ouhsc.edu blooded animals including humans [1]. This pathogen is one of the most common in humans due to many contributing factors that include: (1) its complex life cycle allows it to be transmitted both sexually via felid fecal matter and asexually via carnivorism. (2) Toxoplasma has an extremely wide host cell tropism that includes most nucleated cells. (3) In humans and other intermediate hosts, Toxoplasma develops into a chronic infection that cannot be eliminated by the host's immune response or by currently used drugs. In most cases, chronic infections are largely asymptomatic unless the host becomes immune compromised. Together, these and other properties have allowed Toxoplasma to achieve infection rates that range from $\sim 23 \%$ in the USA [2] to $50-70 \%$ in France [3, 4].

In humans and other intermediate hosts, infections are the result of digesting parasites shed in felid feces or present in undercooked meat [4]. Both infection routes result in the infection of intestinal cells after which the parasites develop into tachyzoites, which are the fastgrowing, disseminating form of the parasite. Tachyzoites replicate within intestinal cells where they stimulate recruitment of neutrophils and dendritic cells. The parasite can then infect these immune cells and use them to disseminate throughout their hosts $[5,6]$. Once parasite reach their target tissue they respond to the resulting IFN $\gamma$ based $\mathrm{T}_{\mathrm{h}} 1$ response by transforming into bradyzoites. Ultimately, bradyzoites will form quiescent tissue cysts that do not cause any significant disease [7]. Bradyzoite conversion is a critical step in the parasite's life cycle since bradyzoites are impervious to immune-mediated destruction, are relatively non-immunogenic, and are the infectious form of the parasite during horizontal transmission (e.g. digestion of undercooked meat). Thus, it is critical that tachyzoites evade IFN $\gamma$-induced death while they convert to bradyzoites. The molecular details underlying each of 
these processes are largely unknown but are important because these data could lead to the development of new drugs to treat the infection.

The past decade has seen important developments in the molecular tools to study Toxoplasma gondii. These include the development of transfection technologies [8-10], sequencing of both host and parasite genomes (see www. toxodb.org), increasing use and refinement of highthroughput genomic and proteomic technologies [11-17], sensitive whole animal imaging [18-20], and large-scale mutagenesis-based screens [21, 22]. These technologies and approaches have been instrumental in increasing our understanding of Toxoplasma replication within its host cell, bradyzoite development, and virulence mechanisms. In this review, we will focus our discussion on how the use of DNA microarrays and other high-throughput transcriptome analysis contributed to these developments and the implications these findings have for ocular disease.

The role of host cell transcription in Toxoplasma gondii growth

A common requirement for intracellular pathogens is they must scavenge nutrients from their hosts while avoiding innate host defense mechanisms [23]. Toxoplasma is no different and how it replicates within a host cell has been the focus of intense investigation by several laboratories. Biochemical- and cell-biological-based assays demonstrated that parasites modify host microtubule and intermediate filament organization [24-26], inhibit host cell apoptosis [27, 28], upregulate pro-inflammatory cytokines [29-32], and scavenge purine nucleosides, cholesterol, and other nutrients from their host cells [33, 34].

To examine the molecular basis for these changes, we and others used DNA microarrays to analyze changes in host gene expression following infection [11, 17, 35]. These studies indicated that changes in host transcription were extremely widespread. These changes came in at least two distinct waves with the first wave being induced within 2 hours and included a large number of pro-inflammatory response genes [11]. The significance of the expression of these genes will be discussed later in this review. Besides the inflammatory response genes, the first wave of gene expression also included genes (EGR1, EGR2, c-jun, and jun-B) that encode transcription factors commonly activated in response to cellular stresses. These data suggests that activation of these genes helps the infected host cell withstand the stress of a Toxoplasma infection. In support of this hypothesis, upregulation of these genes is not a general feature of a cell's response to infection since these genes were not modulated in host cells infected with either Trypanosoma cruzi [36] or the closely related Apicomplexan parasite, Neospora caninum [37]. This result indicated that parasite activation of these transcription factors is accomplished through a Toxoplasma-derived molecule that interacts with a specific host protein. One mechanism by which Toxoplasma can specifically signal to its host cell is by the release of proteins from the rhoptries, which are specialized secretory organelles that contain proteins secreted into the host cytoplasm and nucleus, in a manner analogous to bacterial Type III secretion systems [38]. Consistent with rhoptries being key regulators of host cell functions, upregulation of EGR2 and, likely the other immediate early response host transcription factors, is mediated by a rhoptry factor [37].

The second wave of gene expression included genes that encode proteins that function in a diverse set of cellular processes. Most striking from these studies was the finding that glucose, mevalonate, and iron metabolic genes were upregulated specifically by Toxoplasma [11]. This was intriguing because these genes function in pathways related to some of Toxoplasma's auxotrophies. Thus, their upregulation may be necessary to increase levels these nutrients for the parasite to scavenge. Expression of the glycolytic and iron genes (as well as other genes also observed in the microarray experiments) is regulated by a common transcription factor named hypoxia inducible factor 1 (HIF1) $[39,40]$. HIF1 is a heterodimer composed of $\alpha$ and $\beta$ subunits and stabilization of HIF $1 \alpha$ is the rate-limiting step to its activation. Consistent with the array data, Toxoplasma increased HIF $1 \alpha$ proteins levels, activated HIF1-dependent transcription, and required HIF $1 \alpha$ for parasite growth [41].

Host cell cycle modulation is a common cellular target for many types of pathogens [42]. When both proliferating and non-proliferating cells are infected with Toxoplasma, genes commonly associated with cell growth are differentially modulated $[11,43]$. These data suggested that like other intracellular pathogens, Toxoplasma is actively modulating the host's cell cycle. This hypothesis was tested by several groups who demonstrated that parasite infection leads to changes in host cell cycle progression and causes cells to arrest at the G2/M border [16, 44, 45]. Toxoplasma's effect on the host cell cycle was cell type independent, which was consistent with the microarray data noting differential expression of these genes in various types of infected host cells. Surprisingly, both replicating and senescent cells were similarly affected. The specific parasite factor(s) that regulate the host cell cycle is unknown but at least one appears to be a secreted factor larger than $10 \mathrm{kDa}$ [45]. The importance of such an extrinsic-acting factor is unknown but could be to optimize neighboring cells for infection.

Besides regulating expression of metabolic and cell cycle genes, anti-apoptotic transcripts are a third major class of genes upregulated in Toxoplasma-infected cells. Given that apoptosis of cells infected with viruses and 
bacteria is one host defense against infection [46, 47], it is logical that Toxoplasma would actively prevent host cell apoptosis and appears to do so by interfering with both extrinsic and intrinsic induction of apoptosis (reviewed in [48]). The extrinsic pathway is activated by death signals such as TNF $\alpha$ and FAS and is dependent on Caspase 8. Toxoplasma interferes with this process by blocking Caspase 8 activity [49]. In contrast, parasite modulation of the intrinsic pathway, which is activated by intracellular stress and the subsequent release of cytochrome $\mathrm{C}$ from the mitochondrion, is dependent on the host cell transcription factor NF-KB [50]. NF- $\mathrm{KB}$ is a family of five different proteins and several of these have been observed to be activated in parasite-infected host cells [51-53]. The NF$\mathrm{kB}$-regulated genes required for parasite inhibition of host cell apoptosis are unknown but candidates include the antiapoptotic gene bcl2 as well as several members of the IAP family.

Turning on and off the switches that regulate bradyzoite development

Bradyzoite development is a complex process in which the parasite expresses enzymes that allow it to form a cyst wall while dramatically altering its metabolism and immunological characteristics [7]. These changes are important because bradyzoite-containing cysts are impervious to host defenses and currently prescribed anti-toxoplasmotic drugs. In addition, bradyzoite differentiation is a critical step in the parasite's life cycle since cysts are a transmissible form of the parasite during horizontal transfer. Molecular characterization of the genes encoding bradyzoite-specific antigens indicated that their stage-specific expression was due, in large part, to increasing the abundance of the transcripts that encode them [54-56]. It was not until the transcriptomes of bradyzoites and tachyzoites were directly compared by either comparative EST sequencing or SAGE analysis that the extent of these changes were realized $[57,58]$. Although these studies were critical in allowing us to appreciate the complexity of the transcriptional changes that take place during development, the laborious nature of preparing and analyzing EST and SAGE libraries limited their ability to assess the dynamic nature of bradyzoite development.

In contrast, an important advantage of DNA microarrays is that they can readily examine multiple time points and conditions [59]. As a first step, microarrays spotted with the cDNAs used for the bradyzoite EST sequencing project [58] were generated and used to compare the transcriptional responses that take place at various time points following induction of differentiation [13]. Although these first generation microarrays were spotted with fewer than 650 unique genes, they demonstrated that the microarrays could be used to discover additional bradyzoite-specific genes.
Besides gene discovery, DNA microarrays can also be used to map transcriptional pathways. As an example, the transcriptional response of wild-type parasites and bradyzoite differentiation mutants were compared after stimulating the parasites to undergo differentiation. The resulting microarray data demonstrated that the transcriptional pathways induced during development were hierarchal $[60,61]$.

The full complexity associated with differentiation was demonstrated using full-genome Toxoplasma microarrays that compared the transcriptional responses of three distinct Toxoplasma strains to a drug that induces bradyzoite development [62]. Analysis of the 5' proximal promoters of some bradyzoite-specific genes identified a short $6-8$ bp sequence that conferred stage-specific expression. Electrophoretic mobility shift assays indicated that a parasite nuclear factor binds this promoter element [62]. This was a significant finding because Toxoplasma's genome (and those of other Apicomplexan parasites) lacked genes that shared homology with most known transcription factors. Recently, however, a novel transcription factor family (ApiAP2) was identified in Plasmodium falciparum [63] and subsequent sequence analysis indicated that Toxoplas$m a$ 's genome possesses approximately 40 ApiAP2 family members [64]. Given the lack of other known transcription factors and the large number ApiAP2 proteins, it is likely that one or more members of this family will be involved in bradyzoite development.

Post-translational modifications of histones (e.g. acetylation and methylation) are a widespread epigenetic mechanism critical for regulating gene expression [65]. Due to a lack of experimentally confirmed bradyzoiteinducing transcription factors, investigators began testing whether bradyzoite-specific gene expression could be regulated by epigenetic remodeling. These experiments demonstrated that the promoters of bradyzoite-specific genes in parasites growing under tachyzoite conditions have low levels of acetylated histones and become more extensively modified after exposure to bradyzoite-inducing conditions $[66,67]$.

Histone acetylation is a reversible modification controlled by enzymes that add (histone acetyltransferases (HATs)) or remove (histone deacetylases (HDACs)) acetyl groups from specific lysine residues in the various histones. The importance of histone acetylation was demonstrated by inhibiting HDAC3, a histone H4 deacetylase. Treatment of parasites with a HDAC3 inhibitor (FR235222) induced tachyzoite to bradyzoite differentiation. DNA complexes co-immunoprecipitated using $\alpha$-acetyl-H4 antibodies and hybridized to a high-resolution Toxoplasma DNA microarray demonstrated that the promoters of many but not all bradyzoite genes were hyper-acetylated on histone H4 [68]. But the fact that not all bradyzoite-specific genes harbored this modification suggests that other histone modifications 
may be important for regulating these genes. Altogether, these data paint a picture in which differentiation is controlled transcriptionally by both DNA binding proteins and by epigenetic-based histone modifications. But how all of these changes come together to convert a tachyzoite to a bradyzoite remains unclear.

Besides epigenetic control of Toxoplasma gene expression during bradyzoite differentiation, HATs are expressed in tachyzoites [69, 70] and the expression of some tachyzoite-specific genes are epigenetically regulated [67, 71]. An extremely high-resolution DNA oligonucleotide Toxoplasma microarray representing a well-annotated region of Chromosome $\mathrm{Ib}$ was used in CHIP-on-chip assays to characterize the organization of active and silent promoters in tachyzoites [72]. This study demonstrated that the location and organization of specific modifications of acetylated and methylated histones within the genome could predict not only whether a promoter was active but the $5^{\prime}-3^{\prime}$ orientation of the gene it was regulating.

As an intracellular pathogen, the interplay between the parasite and host cell is likely to have an impact on all aspects of the parasite life cycle including bradyzoite development. An example of this interplay came from the observation that a novel drug named Compound 1 stimulated bradyzoite development in specific Toxoplasma strains [73]. Multifactorial microarray analysis of RNA isolated from Compound-1-treated, mock-infected, or parasite-infected host cells led to the discovery that overexpression of a human gene named CDA1 was sufficient to promote bradyzoite development. CDA1 encodes a protein whose overexpression leads to cell cycle arrest suggesting the status of the host's cell cycle determines if a parasite will undergo bradyzoite development. This is an intriguing hypothesis given the observations that bradyzoites appear to preferentially develop in cells such as neurons and muscle cells that have exited from the cell cycle [74-76].

\section{Virulence}

The population structure of Toxoplasma is extremely clonal and the genotypes of the majority of Toxoplasma strains isolated in North America and Europe group into one of three clonal lineages (types I, II, and III) [77, 78]. In mice, type I strains are highly virulent while the other two are significantly less so [79]. Although all Toxoplasma strain types can cause disease in human infections, type II strains are more commonly associated with congenital infections and toxoplasmic encephalitis while type I and other atypical strains are more commonly associated with postnatally acquired infections that lead to ocular disease [80, 81]. Understanding the basis for differences between Toxoplasma strain types is important for two reasons. First, optimal treatment options to either prevent or cure reactivated infections may be dictated by the parasite's genotype. Second, optimal vaccine design necessitates identifying non-polymorphic antigens.

Toxoplasma virulence is a multi-step, complicated process comprised of transmission, dissemination, host immune evasion, encystation, and reactivation. Although it was commonly accepted that multiple parasite genes would be important for virulence, the first experimental data that this was true came from the finding that a cross between either two avirulent genotypes (types II and III) resulted in virulent progeny [82]. Quantitative trait locus (QTL) mapping of these progeny identified five virulence loci [83]. Thus far, two of these virulence genes have been identified as ROP16 and ROP18. These virulence genes encode rhoptry kinases that are secreted into infected host cells. In an independent study, QTL mapping of progeny from a type I/III cross also identified ROP18 as a virulence gene [84].

One way that the different strains may affect virulence is by differentially modulating host gene expression [12]. Based on human DNA microarray analysis, over 3,000 host genes were differentially expressed in cells infected with progeny from the type II/III cross [12]. Expression QTL mapping of these differences in host gene expression indicated that at least one locus on each parasite chromosome is responsible for differential expression of a portion of these host genes. Modulation of the largest number of these genes $(\sim 1,100$ genes $)$ mapped to a single locus on Chromosome VIIb and ROP16 was determined to be the gene responsible for these differences in host gene expression. Pathway analysis indicated that many ROP16modulated genes were targets of the STAT3/STAT6 transcription factors. How ROP16 specifically regulates STAT3/ STAT6-dependent expression is unknown but infection with parasites harboring the type III allele of ROP16 (which is identical to the allele in type I strains) leads to sustained activation of STAT3/STAT6 [12]. Given ROP16's role in virulence it is therefore tempting to speculate that sustained STAT3/STAT6 activation causes an overproduction of proinflammatory cytokines that can induces immune-mediated tissue destruction.

$\mathrm{NK}$ and T-cell-derived IFN $\gamma$ is the critical cytokine in protection against infections with all Toxoplasma strains [85]. This cytokine protects against Toxoplasma infections by upregulating the expression of inducible nitric oxide synthase, indoleamine dioxygenase, and a family of IFN $\gamma$ regulated GTPases that degrade the parasitophorous vacuole (reviewed in [86]). Regardless of its effectiveness, some parasites can evade IFN $\gamma$-mediated killing and develop into bradyzoites. One possible mechanism by which the parasite avoids IFN $\gamma$ is to disable IFN $\gamma$-induced signaling. Indeed, microarray and cell biological assays demonstrated that IFN $\gamma$-induced transcription is abrogated in cells previously 
infected with Toxoplasma [87, 88]. In contrast to the polymorphic ROP16 and ROP18 virulence factors, Toxoplasma's effects on IFN $\gamma$-dependent transcription are strain independent [87]. The mechanism underlying parasite abrogation of IFN $\gamma$-stimulated transcription is still unclear but does not appear to involve blocking nuclear localization of STAT1, which is a key IFN $\gamma$-regulated transcription factor. However, infection upregulates the expression of members of the suppressor of cytokine signaling (SOCS) family that function by preventing sustained STAT1 activation or levels [89]. Thus, the parasite may utilize a feedback regulatory mechanism to reduce IFN $\gamma$-dependent signaling. It should be noted that others have failed to find evidence of SOCS-mediated inhibition of IFN $\gamma$ signaling since they did not detect differences in STAT1 protein levels in mock- or parasite-infected cells $[87,90]$. Instead, these studies suggest that the parasite affects the ability for STAT1 to bind DNA.

Implications for ocular toxoplasmosis

It is critical that the retina be protected from inflammationinduced damaged because photoreceptors and other neuroretinal cells cannot be replaced or regenerated. One way the retina does this is by maintaining its immuneprivileged state that is characterized by a lack of lymphatics, few endogenous antigen-presenting cells, and the blood-retinal barrier [91]. Regardless, retinal infections do occur and proper regulated protective immune responses are necessary.

Toxoplasma is one of the most common infections of the retina and normally resides in quiescent tissue cysts. Two lines of evidence indicate that in the retina Toxoplasma cysts occasionally reactivate (reviewed in [92]). First, parasite-induced retinal scarring is often observed in Toxoplasma-infected individuals. In many cases, active lesions lie adjacent to older ones and are noted as satellite scars. This satellite scarring is caused by the short migration of some parasites after they emerge from a reactivated cyst. These migrated parasites then re-encyst and the new lesion begins after this cyst reactivates. Second, immune suppression of a chronically infected animal leads to ocular toxoplasmosis. Together, these data indicate that Toxoplas$m a$ tissue cysts are normally found in the retina, they sporadically reactivate, and efficient immune responses are required to limit parasite growth and stimulate them to reencyst.

CD8 T cells, and to a lesser extent CD4 T cells and B cells, are required for protection against Toxoplasma in the peripheral tissues and the retina [93-95]. In addition, the early recruitment of blood-derived monocytes and neutrophils is also critical for protection [96, 97]. Thus, a key question is what signals the recruitment of these cells to the infected retina. DNA microarray analysis of several types of parasite-infected retinal cells (Müller and endothelial cells) [98, 99] demonstrated that several chemokines, whose primary function are the recruitment of immune cells, were upregulated by infection. These chemokines included those that recruit neutrophils-(CXCL1 $(\mathrm{GRO} \alpha)$, CXCL2 (GRO $\beta$ ), CXCL8 (IL-8), and CXCL6 (GCP2)); monocytes-CCL2 (MCP1), CCL5 (RANTES), and CX3CL1 (fractalkine); and T cells-CXCL10 (IP10) and CXCL11 (I-TAC). These data are consistent with the finding that neutrophils, monocytes, and $\mathrm{T}$ cells are the major types of leukocytes present in Toxoplasma-infected retinas (E. Charles and I. J. Blader, manuscript in preparation). These data are also largely consistent with the responses of other types of Toxoplasma-infected host cells suggesting that parasite-induced chemokine expression is largely independent of the type of infected host cell or tissue.

While these experiments have helped shed light on what directs immune cells to parasite-infected retinas, they still leave many questions unanswered. First, how are reactivated infections recognized in the retina? In the periphery, various TLR receptors (e.g. TLR2, TLR4, and TLR11) recognize Toxoplasma-derived factors [100-102]. But whether specific TLRs or other pattern recognition molecules are required in the retina is unclear [103]. While it is established that toll receptors are expressed in the retina it is not known how they contribute to the mobilization of a memory T-cell-based retinal immune response [104, 105]. Second, which antigen presenting cells are responsible for activation of memory $\mathrm{CD}^{+}$and $\mathrm{CD} 4^{+} \mathrm{T}$ cells? This is a particularly interesting problem for the retina since the existence of professional antigen-presenting cells in the retina has not been formally established. Thus, does infection induce a resident retinal cell (e.g. retinal microglia or astrocyte) to develop these properties or is antigen presentation a function only fulfilled by an infiltrating leukocyte? Third, which type of memory (effector vs. central) $\mathrm{T}$ cell is activated during a reactivated retinal infection? Finally, do the $\mathrm{T}$ cells that gain access to the infected retina do so because of a breach in blood-retinal barrier integrity or because the retina selectively permits specific types of $\mathrm{T}$ cells to gain entry? Obviously, these issues are not unique to ocular toxoplasmosis but are also relevant to other types of infection of the retina and other immune-privileged tissues. But given the experimental manipulability of Toxoplasma, it is likely that important progress will soon be made.

In addition to the immunological aspects of ocular toxoplasmosis, more parasite-centric questions regarding ocular toxoplasmosis need to be addressed and DNA microarrays are poised to have an important role in addressing them. For example, what parasite virulence factors act in the retina? As discussed above, ROP16 and 
ROP18 were identified as virulence factors using QTLbased approaches. But, how these two proteins function is not known nor is it clear whether they would have a role in the retina. Besides these two proteins, the SAG1 parasite surface protein was proposed to have an important role in Toxoplasma-induced intestinal damage and loss of SAG1 had a slight effect on subsequent retinal damage after parasites were injected into the anterior chamber of the eye $[106,107]$. But, when parasites were directly injected into the posterior chamber, there were no apparent differences in retinal damage between wild-type and SAG1 knockout parasites [108]. These data suggested that SAG1 was not acting as a virulence factor per se in the retina but rather may help the parasite cross anatomical barriers (e.g. traffic from the anterior to posterior chambers).

Another question that has not been addressed is how parasite gene expression is influenced by the host cell it is growing within. This could be important since different host cells present different environments and challenges that the parasite must overcome. For example, macrophages may release higher levels and be more responsive to antitoxoplasmotic host defense molecules such as oxygen radicals, IFN $\gamma$, and TNF $\alpha$. To survive in these conditions, parasites have developed several strategies. For example, they express an efficient set of antioxidant proteins that protect the parasite from oxidative damage [109]. But the parasite also express novel stress response/immune evasion factors as was demonstrated by the discovery that a novel parasite protein containing a patatin-like phospholipase domain was required for survival in activated macrophages [110]. It is likely that additional stress response/immune evasion factors remain to be discovered. As they have in bacterial and fungal pathogens, DNA microarrays are poised to have an important role in the identification of these factors and the proteins they interact with [111, 112].

As a parasite with a potentially devastating clinical outcome, an important goal of ocular toxoplasmosis research is the development of new drugs and treatments. There are two major reasons that new drugs are needed to treat Toxoplasma infections. First, the drugs currently used to treat Toxoplasma infections are poorly tolerated, have severe side effects, and cannot act against bradyzoites $[113,114]$. Second, there are reports that Toxoplasma is developing resistance to the current generation of drugs $[115,116]$. How resistance to these drugs has developed is not known but is critical to understand because it will lead to improved drug design and will increase our understanding of the biological functions of these drug targets. One way to understanding mechanisms of resistance is to compare the transcriptional profiles of wild-type and resistant parasites grown in the absence or presence of the drug. Such studies in bacterial resistance have demonstrated that pathogen responses to antibiotics are multifactorial and complex [117]. Whether the same will be true in Toxoplasma is unclear, but data from these types of experiments will likely impact new anti-Toxoplasma drug design.

Ocular toxoplasmosis is more severe and prevalent in Brazil than in Europe or North America suggesting that either parasite strains or host responses are responsible for these differences [118]. Genotyping of strains from the Brazilian patients have identified parasite strains that are highly divergent from the types I, II, and III strains found in Europe and North America [119]. But what properties of these strains confer their ability to cause severe ocular disease is not known. Given the significantly reduced costs of whole genome sequencing, it is likely that genome sequences of these novel strains will provide important clues. But, these studies will need to be followed by others that examine the transcriptome and proteome of these strains in order to understand how these changes in sequence correspond to biologically distinct phenotypes.

\section{Summary}

Over the past decade, the application of host and parasite microarrays have allowed Toxoplasma researchers to make great strides in understanding how Toxoplasma grows, differentiates, and causes disease. The majority of these experiments have thus far focused on tissue culture-based experimental systems or death-as-endpoint virulence studies. Relative to these systems, our understanding of how Toxoplasma interacts with and causes disease in the retina has been lagging. But the techniques and technologies that these other studies have pioneered (e.g. microarrays, QTL screening, and epigenetic mapping) coupled with highthroughput DNA sequencing and proteomics, will allow ocular toxoplasmosis researchers to make important and rapid advances in the very near future.

Acknowledgements We thank Dr. Bill Sullivan for his critical reading of this review. Work in our laboratory is supported by grants from the National Institutes of Health/NIAID (RO1AI069986) and the American Cancer Society (MBC-114461).

Open Access This article is distributed under the terms of the Creative Commons Attribution Noncommercial License which permits any noncommercial use, distribution, and reproduction in any medium, provided the original author(s) and source are credited.

\section{References}

1. Montoya JG, Liesenfeld O. Toxoplasmosis. Lancet. 2004;363 (9425):1965-76.

2. Jones JL, et al. Toxoplasma gondii infection in the United States: seroprevalence and risk factors. Am J Epidemiol. 2001;154 (4):357-65. 
3. Jeannel D, et al. Epidemiology of toxoplasmosis among pregnant women in the Paris area. Int J Epidemiol. 1988;17(3):595-602.

4. Tenter AM, Heckeroth AR, Weiss LM. Toxoplasma gondii: from animals to humans. Int J Parasitol. 2000;30(12-13):1217-58.

5. Lambert $\mathrm{H}$, et al. Induction of dendritic cell migration upon Toxoplasma gondii infection potentiates parasite dissemination. Cell Microbiol. 2006;8(10):1611-23.

6. Courret N, et al. CD11c- and CD11b-expressing mouse leukocytes transport single Toxoplasma gondii tachyzoites to the brain. Blood. 2006;107(1):309-16.

7. Weiss LM, Kim K. The development and biology of bradyzoites of Toxoplasma gondii. Front Biosci. 2000;5:D391-405.

8. Soldati D, Boothroyd JC. Transient transfection and expression in the obligate intracellular parasite Toxoplasma gondii. Science. 1993;260(5106):349-52.

9. Donald RG, Roos DS. Stable molecular transformation of Toxoplasma gondii: a selectable dihydrofolate reductasethymidylate synthase marker based on drug-resistance mutations in malaria. Proc Natl Acad Sci U S A. 1993;90 (24):11703-7.

10. Sibley LD, Messina M, Niesman IR. Stable DNA transformation in the obligate intracellular parasite Toxoplasma gondii by complementation of tryptophan auxotrophy. Proc Natl Acad Sci U S A. 1994;91(12):5508-12.

11. Blader IJ, Manger ID, Boothroyd JC. Microarray analysis reveals previously unknown changes in Toxoplasma gondii-infected human cells. J Biol Chem. 2001;276(26):24223-31.

12. Saeij JP, et al. Toxoplasma co-opts host gene expression by injection of a polymorphic kinase homologue. Nature. 2007;445 (7125):324-7.

13. Cleary MD, et al. Toxoplasma gondii asexual development: identification of developmentally regulated genes and distinct patterns of gene expression. Eukaryotic Cell. 2002;1(3):329-40.

14. Xia D, et al. The proteome of Toxoplasma gondii: integration with the genome provides novel insights into gene expression and annotation. Genome Biol. 2008;9(7):R116.

15. Bradley PJ, et al. Proteomic analysis of rhoptry organelles reveals many novel constituents for host-parasite interactions in Toxoplasma gondii. J Biol Chem. 2005;280(40):34245-58.

16. Nelson MM, et al. Modulation of the host cell proteome by the intracellular apicomplexan parasite Toxoplasma gondii. Infect Immun. 2008;76(2):828-44.

17. Chaussabel D, et al. Unique gene expression profiles of human macrophages and dendritic cells to phylogenetically distinct parasites. Blood. 2003;102(2):672-81.

18. Saeij JP, et al. Bioluminescence imaging of Toxoplasma gondii infection in living mice reveals dramatic differences between strains. Infect Immun. 2005;73:695-702.

19. Hitziger $\mathrm{N}$, et al. Dissemination of Toxoplasma gondii to immunoprivileged organs and role of Toll/interleukin-1 receptor signalling for host resistance assessed by in vivo bioluminescence imaging. Cell Microbiol. 2005;7(6):837-48.

20. Dellacasa-Lindberg I, Hitziger N, Barragan A. Localized recrudescence of Toxoplasma infections in the central nervous system of immunocompromised mice assessed by in vivo bioluminescence imaging. Microbes Infect. 2007;9(11):12918 .

21. Frankel MB, Mordue DG, Knoll LJ. Discovery of parasite virulence genes reveals a unique regulator of chromosome condensation 1 ortholog critical for efficient nuclear trafficking. Proc Natl Acad Sci U S A. 2007;104(24):10181-6.

22. Gubbels MJ, et al. Forward genetic analysis of the apicomplexan cell division cycle in Toxoplasma gondii. PLoS Pathog. 2008;4 (2):e36.

23. Sinai AP, Joiner KA. Safe haven: the cell biology of nonfusogenic pathogen vacuoles. Annu Rev Microbiol. 1997;51:415-62.
24. Coppens I, et al. Toxoplasma gondii sequesters lysosomes from mammalian hosts in the vacuolar space. Cell. 2006;125(2):26174

25. Walker ME, et al. Toxoplasma gondii actively remodels the microtubule network in host cells. Microbes Infect. 2008;10(14 15):1440-9.

26. Halonen SK, Weidner E. Overcoating of Toxoplasma parasitophorous vacuoles with host cell vimentin type intermediate filaments. J Eukaryot Microbiol. 1994;41(1):65-71.

27. Nash PB, et al. Toxoplasma gondii-infected cells are resistant to multiple inducers of apoptosis. J Immunol. 1998;160(4):1824 30 .

28. Goebel S, Luder CG, Gross U. Invasion by Toxoplasma gondii protects human-derived HL-60 cells from actinomycin Dinduced apoptosis. Med Microbiol Immunol (Berl). 1999;187 (4):221-6

29. Li ZY, et al. Toxoplasma gondii soluble antigen induces a subset of lipopolysaccharide-inducible genes and tyrosine phosphoproteins in peritoneal macrophages. Infect Immun. 1994;62 (8):3434-40.

30. Brenier-Pinchart MP, et al. Toxoplasma gondii induces the secretion of monocyte chemotactic protein-1 in human fibroblasts, in vitro. Mol Cell Biochem. 2000;209(1-2):79-87.

31. Yap GS, Sher A. Cell-mediated immunity to Toxoplasma gondii: initiation, regulation and effector function. Immunobiology. 1999;201(2):240-7.

32. Denney CF, Eckmann L, Reed SL. Chemokine secretion of human cells in response to Toxoplasma gondii infection. Infect Immun. 1999;67(4):1547-52.

33. Schwartzman JD, Pfefferkorn ER. Toxoplasma gondii: purine synthesis and salvage in mutant host cells and parasites. Exp Parasitol. 1982;53(1):77-86.

34. Coppens I, Sinai AP, Joiner KA. Toxoplasma gondii exploits host low-density lipoprotein receptor-mediated endocytosis for cholesterol acquisition. J Cell Biol. 2000;149(1):167-80.

35. Gail M, Gross U, Bohne W. Transcriptional profile of Toxoplasma gondii-infected human fibroblasts as revealed by gene-array hybridization. Mol Genet Genomics. 2001;265(5):905-12.

36. de Avalos SV, et al. Immediate/early response to Trypanosoma cruzi infection involves minimal modulation of host cell transcription. J Biol Chem. 2002;277(1):639-44.

37. Phelps E, Sweeney K, Blader IJ. Toxoplasma gondii rhoptry discharge correlates with activation of the EGR2 host cell transcription factor. Infect Immun. 2008;76(10):4703-12.

38. Gilbert LA, et al. Toxoplasma gondii targets a protein phosphatase $2 \mathrm{C}$ to the nuclei of infected host cells. Eukaryot Cell. 2007;6 (1):73-83.

39. Semenza GL. Hypoxia-inducible factor 1 (HIF-1) pathway. Sci STKE. 2007;2007(407):cm8.

40. Zinkernagel AS, Johnson RS, Nizet V. Hypoxia inducible factor (HIF) function in innate immunity and infection. J Mol Med. 2007;85(12):1339-46.

41. Spear W, et al. The host cell transcription factor hypoxiainducible factor 1 is required for Toxoplasma gondii growth and survival at physiological oxygen levels. Cell Microbiol. 2006;8 (2):339-52.

42. Galan JE, Cossart P. Host-pathogen interactions: a diversity of themes, a variety of molecular machines. Curr Opin Microbiol. 2005;8(1):1-3.

43. Molestina RE, Sinai AP. Host and parasite-derived IKK activities direct distinct temporal phases of NF-kappaB activation and target gene expression following Toxoplasma gondii infection. J Cell Sci. 2005;118(Pt 24):5785-96.

44. Brunet J, et al. Toxoplasma gondii exploits UHRF1 and induces host cell cycle arrest at G2 to enable its proliferation. Cell Microbiol. 2008;10(4):908-20. 
45. Garrison EM, Arrizabalaga G. Disruption of a mitochondrial MutS DNA repair enzyme homologue confers drug resistance in the parasite Toxoplasma gondii. Mol Microbiol. 2009;72(2):42541.

46. Roulston A, Marcellus RC, Branton PE. Viruses and apoptosis. Annu Rev Microbiol. 1999;53:577-628.

47. Faherty CS, Maurelli AT. Staying alive: bacterial inhibition of apoptosis during infection. Trends Microbiol. 2008;16(4):173-80.

48. Sinai AP, et al. Mechanisms underlying the manipulation of host apoptotic pathways by Toxoplasma gondii. Int J Parasitol. 2004;34(3):381-91.

49. Vutova P, et al. Toxoplasma gondii inhibits Fas/CD95-triggered cell death by inducing aberrant processing and degradation of caspase 8. Cell Microbiol. 2007;9(6):1556-70.

50. Payne TM, Molestina RE, Sinai AP. Inhibition of caspase activation and a requirement for NF- $\{$ kappa $\}$ B function in the Toxoplasma gondii-mediated blockade of host apoptosis. J Cell Sci. 2003;116(Pt 21):4345-58.

51. Molestina RE, et al. Activation of NF- $\{$ kappa $\}$ B by Toxoplasma gondii correlates with increased expression of antiapoptotic genes and localization of phosphorylated I\{kappa\}B to the parasitophorous vacuole membrane. J Cell Sci. 2003;116(Pt 21):4359-71.

52. Kim JM, et al. Nuclear factor-kappa B plays a major role in the regulation of chemokine expression of HeLa cells in response to Toxoplasma gondii infection. Parasitol Res. 2001;87(9):758-63.

53. Shapira $\mathrm{S}$, et al. Initiation and termination of NF-kappaB signaling by the intracellular protozoan parasite Toxoplasma gondii. J Cell Sci. 2005;118(Pt 15):3501-8.

54. Yang S, Parmley SF. A bradyzoite stage-specifically expressed gene of Toxoplasma gondii encodes a polypeptide homologous to lactate dehydrogenase. Mol Biochem Parasitol. 1995;73(1-2):291-4.

55. Bohne W, et al. Cloning and characterization of a bradyzoitespecifically expressed gene (hsp30/bag1) of Toxoplasma gondii, related to genes encoding small heat-shock proteins of plants. Mol Microbiol. 1995;16(6):1221-30.

56. Odberg-Ferragut $\mathrm{C}$, et al. Molecular cloning of the Toxoplasma gondii sag4 gene encoding an $18 \mathrm{kDa}$ bradyzoite specific surface protein. Mol Biochem Parasitol. 1996;82(2):237-44.

57. Radke JR, et al. The transcriptome of Toxoplasma gondii. BMC Biol. 2005;3:26.

58. Manger ID, et al. Expressed sequence tag analysis of the bradyzoite stage of Toxoplasma gondii: identification of developmentally regulated genes. Infect Immun. 1998;66(4):1632-7.

59. Boothroyd JC, et al. DNA microarrays in parasitology: strengths and limitations. Trends Parasitol. 2003;19(10):470-6.

60. Matrajt $\mathrm{M}$, et al. Identification and characterization of differentiation mutants in the protozoan parasite Toxoplasma gondii. Mol Microbiol. 2002;44(3):735-47.

61. Singh U, Brewer JL, Boothroyd JC. Genetic analysis of tachyzoite to bradyzoite differentiation mutants in Toxoplasma gondii reveals a hierarchy of gene induction. Mol Microbiol. 2002;44(3):721-33.

62. Behnke MS, et al. The transcription of bradyzoite genes in Toxoplasma gondii is controlled by autonomous promoter elements. Mol Microbiol. 2008;68(6):1502-18.

63. De Silva EK, et al. Specific DNA-binding by apicomplexan AP2 transcription factors. Proc Natl Acad Sci U S A. 2008;105 (24):8393-8.

64. Kim K (2008) Using epigenomics to understand gene expression in Toxoplasma tachyzoites, in 10th International Workshops on Opportunistic Protists. Boston, MA

65. Shahbazian MD, Grunstein M. Functions of site-specific histone acetylation and deacetylation. Annu Rev Biochem. 2007;76 (1):75-100.
66. Sullivan WJ Jr, Smith AT, Joyce BR. Understanding mechanisms and the role of differentiation in pathogenesis of Toxoplasma gondii: a review. Mem Inst Oswaldo Cruz. 2009; 104(2):155-61.

67. Saksouk N, et al. Histone-modifying complexes regulate gene expression pertinent to the differentiation of the protozoan parasite Toxoplasma gondii. Mol Cell Biol. 2005;25(23): 10301-14.

68. Bougdour A, et al. Drug inhibition of HDAC3 and epigenetic control of differentiation in Apicomplexa parasites. J Exp Med. 2009;206(4):953-66.

69. Sullivan WJ Jr, Smith CK. 2nd, Cloning and characterization of a novel histone acetyltransferase homologue from the protozoan parasite Toxoplasma gondii reveals a distinct GCN5 family member. Gene. 2000;242(1-2):193-200.

70. Smith AT, et al. MYST family histone acetyltransferases in the protozoan parasite Toxoplasma gondii. Eukaryotic Cell. 2005;4 (12):2057-65.

71. Bhatti MM, et al. Pair of unusual GCN5 histone acetyltransferases and ADA2 homologues in the protozoan parasite Toxoplasma gondii. Eukaryotic Cell. 2006;5(1):62-76.

72. Gissot M, et al. Epigenomic modifications predict active promoters and gene structure in Toxoplasma gondii. PLoS Pathog. 2007;3(6):e77.

73. Radke JR, et al. Changes in the expression of human cell division autoantigen-1 influence Toxoplasma gondii growth and development. PLoS Pathog. 2006;2(10):e105.

74. Guimaraes EV, de Carvalho L, Barbosa HS. Primary culture of skeletal muscle cells as a model for studies of Toxoplasma gondii cystogenesis. J Parasitol. 2008;94(1):72-83.

75. Luder CG, et al. Toxoplasma gondii in primary rat CNS cells: differential contribution of neurons, astrocytes, and microglial cells for the intracerebral development and stage differentiation. Exp Parasitol. 1999;93(1):23-32.

76. Ferreira-da-Silva MD, et al. Primary skeletal muscle cells trigger spontaneous Toxoplasma gondii tachyzoite-to-bradyzoite conversion at higher rates than fibroblasts. Int $\mathrm{J}$ Med Microbiol. 2008;299(5):381-8.

77. Howe DK, Sibley LD. Toxoplasma gondii comprises three clonal lineages: correlation of parasite genotype with human disease. $\mathrm{J}$ Infect Dis. 1995;172(6):1561-6.

78. Darde ML, Bouteille B, Pestre-Alexandre M. Isoenzyme analysis of 35 Toxoplasma gondii isolates and the biological and epidemiological implications. J Parasitol. 1992;78 (5):786-94.

79. Sibley LD, Boothroyd JC. Virulent strains of Toxoplasma gondii comprise a single clonal lineage. Nature. 1992;359(6390):82-5.

80. Bottos J, et al. Bilateral retinochoroiditis caused by an atypical strain of Toxoplasma gondii. Br J Ophthalmol. 2009;93 (11):1546-50.

81. Boothroyd JC, Grigg ME. Population biology of Toxoplasma gondii and its relevance to human infection: do different strains cause different disease? Curr Opin Microbiol. 2002;5(4):438-42.

82. Grigg ME, et al. Success and virulence in Toxoplasma as the result of sexual recombination between two distinct ancestries. Science. 2001;294(5540):161-5.

83. Saeij JP, et al. Polymorphic secreted kinases are key virulence factors in toxoplasmosis. Science. 2006;314(5806):1780-3.

84. Taylor S, et al. A secreted serine-threonine kinase determines virulence in the eukaryotic pathogen Toxoplasma gondii. Science. 2006;314(5806):1776-80.

85. Gaddi PJ, Yap GS. Cytokine regulation of immunopathology in toxoplasmosis. Immunol Cell Biol. 2007;85(2):155-9.

86. Blader IJ, Saeij JP. Communication between Toxoplasma gondii and its host: impact on parasite growth, development, immune evasion, and virulence. APMIS. 2009;117(5-6):458-76. 
87. Kim SK, Fouts AE, Boothroyd JC. Toxoplasma gondii dysregulates IFN-gamma-inducible gene expression in human fibroblasts: insights from a genome-wide transcriptional profiling. J Immunol. 2007;178(8):5154-65.

88. Luder CG, et al. Toxoplasma gondii down-regulates MHC class II gene expression and antigen presentation by murine macrophages via interference with nuclear translocation of STAT1alpha. Eur J Immunol. 2001;31(5):1475-84.

89. Zimmermann $\mathrm{S}$, et al. Induction of suppressor of cytokine signaling-1 by Toxoplasma gondii contributes to immune evasion in macrophages by blocking IFN-gamma signaling. J Immunol. 2006;176(3):1840-7.

90. Lang C, et al. Diverse mechanisms employed by Toxoplasma gondii to inhibit IFN-gamma-induced major histocompatibility complex class II gene expression. Microbes Infect. 2006;8 (8):1994-2005.

91. Streilein JW. Ocular immune privilege: the eye takes a dim but practical view of immunity and inflammation. J Leukoc Biol. 2003;74(2):179-85.

92. Jones LA, Alexander J, Roberts CW. Ocular toxoplasmosis: in the storm of the eye. Parasite Immunol. 2006;28(12):635-42.

93. Liesenfeld $\mathrm{O}$, et al. Association of CD4+ T cell-dependent, interferon-gamma-mediated necrosis of the small intestine with genetic susceptibility of mice to peroral infection with Toxoplasma gondii. J Exp Med. 1996;184(2):597-607.

94. Lu F, Huang S, Kasper LH. CD4+ T cells in the pathogenesis of murine ocular toxoplasmosis. Infect Immun. 2004;72(9):4966-72.

95. Gazzinelli RT, et al. Synergistic role of CD4+ and CD8+ T lymphocytes in IFN-gamma production and protective immunity induced by an attenuated Toxoplasma gondii vaccine. J Immunol. 1991;146(1):286-92.

96. Robben PM, et al. Recruitment of Gr-1+ monocytes is essential for control of acute toxoplasmosis. J Exp Med. 2005;201 (11):1761-9.

97. Del Rio L, et al. CXCR2 deficiency confers impaired neutrophil recruitment and increased susceptibility during Toxoplasma gondii infection. J Immunol. 2001;167(11):6503-9.

98. Smith JR, et al. Unique gene expression profiles of donormatched human retinal and choroidal vascular endothelial cells. Invest Ophthalmol Vis Sci. 2007;48(6):2676-84.

99. Knight BC, et al. The effect of Toxoplasma gondii infection on expression of chemokines by rat retinal vascular endothelial cells. J Neuroimmunol. 2005;160(1-2):41-7.

100. Yarovinsky F, et al. TLR11 activation of dendritic cells by a protozoan profilin-like protein. Science. 2005;308(5728):1626-9.

101. Mun HS, et al. Toll-like receptor 4 mediates tolerance in macrophages stimulated with Toxoplasma gondii-derived heat shock protein 70. Infect Immun. 2005;73(8):4634-42.

102. Debierre-Grockiego F, et al. Activation of TLR 2 and TLR 4 by glycosylphosphatidylinositols derived from Toxoplasma gondii. J Immunol. 2007;179(2):1129-37.

103. Sukhumavasi W, et al. TLR adaptor MyD88 is essential for pathogen control during oral Toxoplasma gondii infection but not adaptive immunity induced by a vaccine strain of the parasite. $\mathrm{J}$ Immunol. 2008;181(5):3464-73.

104. Jiang G, et al. Regulatory role of TLR ligands on the activation of autoreactive T Cells by retinal astrocytes. Invest Ophthalmol Vis Sci. 2009;50(10):4769-76.

105. Yang Z, et al. Toll-like receptor 3 and geographic atrophy in agerelated macular degeneration. N Engl J Med. 2008;359 (14):1456-63.

106. Rachinel N, et al. The induction of acute ileitis by a single microbial antigen of Toxoplasma gondii. J Immunol. 2004;173 (4):2725-35.

107. Lu F, et al. Experimental ocular toxoplasmosis in genetically susceptible and resistant mice. Infect Immun. 2005;73 (8):5160-5.

108. Charles E, Callegan MC, Blader IJ. The SAG1 toxoplasma surface protein is not required for acute ocular toxoplasmosis in mice. Infect Immun. 2007;75:2079-83.

109. Ding M, et al. The antioxidant systems in Toxoplasma gondii and the role of cytosolic catalase in defence against oxidative injury. Mol Microbiol. 2004;51(1):47-61.

110. Mordue DG, et al. A patatin-like protein protects Toxoplasma gondii from degradation in activated macrophages. Mol Microbiol. 2007;63(2):482-96.

111. Gancz H, Censini S, Merrell DS. Iron and $\mathrm{pH}$ homeostasis intersect at the level of Fur regulation in the gastric pathogen Helicobacter pylori. Infect Immun. 2006;74(1):602-14.

112. Jung $\mathrm{WH}$, et al. Iron regulation of the major virulence factors in the AIDS-associated pathogen Cryptococcus neoformans. PLoS Biol. 2006;4(12):e410.

113. Dannemann B, et al. Treatment of toxoplasmic encephalitis in patients with AIDS. A randomized trial comparing pyrimethamine plus clindamycin to pyrimethamine plus sulfadiazine. The California Collaborative Treatment Group. Ann Intern Med. 1992;116(1):33-43.

114. Mccabe R. Antitoxoplasma chemotherapy. In: Joynson DH, Wreghitt TG, editors. Toxoplasmosis: a comprehensive clinical guide. Cambridge: Cambridge University Press; 2001. p. 319 59.

115. Baatz H, et al. Reactivation of toxoplasma retinochoroiditis under atovaquone therapy in an immunocompetent patient. Ocul Immunol Inflamm. 2006;14(3):185-7.

116. Aspinall TV, et al. The molecular basis of sulfonamide resistance in Toxoplasma gondii and implications for the clinical management of toxoplasmosis. J Infect Dis. 2002;185(11):1637-43.

117. Brazas MD, Hancock RE. Using microarray gene signatures to elucidate mechanisms of antibiotic action and resistance. Drug Discov Today. 2005;10(18):1245-52.

118. Claudio S, et al. A follow-up study of Toxoplasma gondii infection in southern Brazil. Am J Ophthalmol. 2001;131 (3):351-4.

119. Khan A, et al. Genetic divergence of Toxoplasma gondii strains associated with ocular toxoplasmosis, Brazil. Emerg Infect Dis. 2006;12(6):942-9. 